
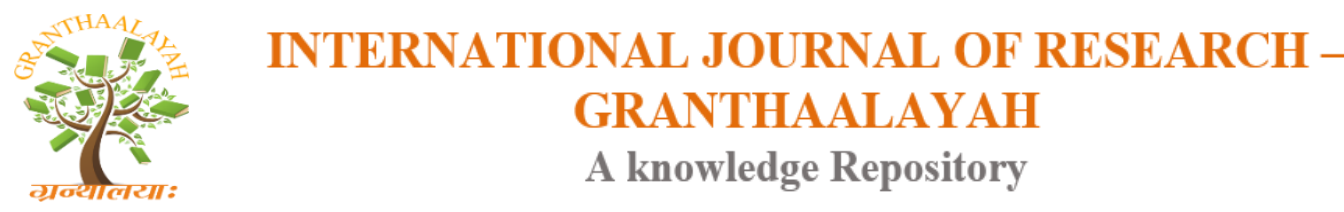

Science

\title{
AYURVEDIC APPROACH FOR ASCITES: A CASE STUDY
}

\author{
Dr. Jain Ankita Pravin 1, Dr. Vivek S. Chandurkar ${ }^{2}$ \\ ${ }^{* 1}$ MD (Scholar) kayachikitsa department, SGR Ayurved Collage Solapur, India \\ ${ }^{2}$ Professor and HOD of Kayachikitsa deparmrnt, SGR Ayurved Collage Solapur, India
}

\begin{abstract}
A 49yrs male patient presenting with udarvriddhi (abdominal distention), dourbalya (gen. weakness), ubhaypadshoth (bilateral pedal edema) diagnosed as Udarvyadhi (ascites) was brought to SSNJ Ayurved Hospital, Solapur.Patient was treated with an integrated approach of ayurveda. According to ayurveda, treatment of is nityavirechana (purgative), agnideepan (increase appetite), balaprapti (stimulant for hepatic function), lepachikitsa, Proper diet (cow milk) and external application of arkapattabandhan (belt made by leaves of Calotropisprocera). Appreciable results were observed in the form of reduction in abdominal girth, decreased pedal edema, increased appetite, increased strength and significant changes in investigations.
\end{abstract}

Keywords: Ayurveda; Yakrut; Udara; Ascites; Virechana; Lepa.

Cite This Article: Dr. Jain Ankita Pravin, and Dr. Vivek S. Chandurkar. (2018). "AYURVEDIC APPROACH FOR ASCITES: A CASE STUDY." International Journal of Research Granthaalayah, 6(9), 321-325. https://doi.org/10.5281/zenodo.1443491.

\section{Introduction}

Ascites is accumulation of fluid in the peritoneal cavity. Ascites is caused very commonly due to cirrhosis and severe liver disease. Sometimes the fluid buildup is mild and cannot be detected externally. But in extreme cases, the fluid builds up to such a large extent that it causes severe abdominal distention. The condition is quite painful and the person suffering from it finds it extremely inconvenient to even move around. Ascites itself is a symptom of several serious problems. The presence of ascites may indicate portalhypertension, hepatitis, heart failure, pericarditis and even cancers. People who consume excessive alcohol are at very high risk of developing cirrhosis, which may cause ascites. According to ayurveda main causes for udarvyadhi are mandagni and garvisha like atimadyapan. Ayurvedic managemant includes oral medications aswell as virechan for specific treatment to reduce accumulation of fluid, as well as treatment to remove the obstruction. Ksheerpan for diet regulation portant part of management of this diseasae. Ayurvedic line of treatment for ascites is mainly virechan. It is useful in cases cites as it has laxative and diuretic action which helps to excrete excess fluid out of body. 


\section{A Case Report}

A 49 yrs male Hindu patient brought by relatives to Seth Sakharam Nemchand Ayurved Hospital, Solapur belonging to the middle socio economic class presenting with complaints of Udaravridhi (increased abdominal girth), Kshudhamandhya (decreasedappetite),Dourbalya(generalweakness), Ubhayapadashotha and Krishnavarna(bilateral pedal oedema and discolouration) from 6 months.

\section{On Examination}

1) General condition of patient is moderate

2) Pulse rate: $78 / \mathrm{min}$

3) B.P. $130 / 80 \mathrm{~mm}$ of $\mathrm{Hg}$

4) Pallor ++

5) icterus +

6) Weight- $72 \mathrm{~kg}$, Height- $164 \mathrm{~cm}$

7) $\mathrm{RS}-\mathrm{AE}=\mathrm{BE}$ clear CVS - S1 S2 normal. no abnormal sound CNS - well conscious oriented

8) P/A - Abdomen was distended with bulging of flanks.

Veins on the wall appears prominent.

Umbilicus transverse.

On palpation liver enlarged.

Shifting dullness present.

9) Bipedal pitting edema present.

\section{Ashtavidhpariksha}

1) Nadi - Vatpradhanpitta

2) Mala - malavshtambha (occasional)

3) Mutra -4 to 5 times/day

4) Jivha -sama

5) Shabd - spashta

6) Sparsh - ushana

7) Druk - panduta

8) Akriti - madhyam

Srotodushti:Rasavahasrotas, Annavahasrotas, Udakvahasritas, Pranavahasrotas.

\section{Investigations}

1) $\mathrm{CBC}$

2) LFT

3) RFT

4) Urine analysis

5) USG abdomen

\section{Past History}

No H/O of DM, HTN, T.B, Asthma or any major illness.

No H/O of any Surgery. 
$\mathrm{H} / \mathrm{O}$ of Alcohol intake for $20 \mathrm{yrs}$ left since 3 months.

\section{Treatment Approach}

1) ArogyavardhiniVati $500 \mathrm{mg}$ (thrice daily).

2) Trivruttavaleha 10 gram with TriphalaKwatha $20 \mathrm{ml}$ (once at night).

3) Punarnavasava $20 \mathrm{ml}$ (twice daily).

4) Tb. SuvarnaSutshekhar 500 mg with Madhu (twice daily).

5) RohitakGhrita $10 \mathrm{ml}$ (twice daily)

6) ShothaharaLepa apply on both leg (Oedematous)

7) Arkapatrawith eranda tail bandhan over abdomen.

8) Patient is only on cow milk.

\section{Discussion}

Patient was treated with an integrated approach of ayuvedic treatment. According to ayurveda treatment of udaris nityavirechana (purgative), agnideepan (increase appetite), balaprapti (increase strength), yakrituttejjak (stimulant for hepatic function) andexternal application of arkapattbandhan (belt made by leaves of Calotropisprocera). Appreciable results were observed in the form of reduction in abdominal girth, decreased pedal edema, increased appetite, and increased strength. Chikitsasidhanth for udarvyadhiis 'nityamevvirechayet'. Virechanacheckes improper jatharagni and dhatvagni, after virechanajatharagni and dhatvagni increases ${ }^{1}$.It has laxative action which helps to eliminate toxinsout of the body, which is caused due tochronic constipation in ascites $^{2}$.It possesses Cholagogue, hepatoprotective and liver stimulant action. Therefore it is useful in generalized oedema and ascites as it has laxative and diuretic action which helps to excrete excess fluid out of body. Arogyavardhini vati acts as yakrituttejak, hepatoprotective activity ${ }^{3}$.Trivrittavleha has action of virechana prominently and isused in ascites cases. It is a bowel regulatorin chronic constipation and induces therapeutic mutral(diuretic) as well as shothaghna (reduces edema) and it purgation by its ushnatikshnavyavayi gunas. Punarnavasava acts as improves renal function. Arkapatrapattabandhan avoids vataprakop by mriduswedan and is supportiveto diuretic action. Cow milk gives strength to the patient without increasing body fluid level in the body. Udar is asadhyavyadhi(incurable) as per ayurveda but we could give symptomatic relief, reduction in fluid, improvement in quality of life to the patient.

\section{Conclusion}

The pathological factors responsible for udarvyadhi are tridosha and reduced status of agni. Virechana is unique treatment mentioned for udar. Removal of doshas mainly pitta and normalize yakritdushti which was caused due to atimadyapan was achieved by this integrated approach of ayurvedic treatment successfully. By this line of treatment there is significant improvement in abdominalgirth, appetite, strength. There is also significant improvement in laboratory findings. So ayurveda can play very important role in treating patients of ascites.

Table 1: ABDOMINAL GIRTH

\begin{tabular}{|l|l|}
\hline Date & At Umbilicus (in cm) \\
\hline $23 / 3 / 2018$ & 98 \\
\hline $24 / 3 / 2018$ & 97 \\
\hline
\end{tabular}




\begin{tabular}{|l|l|}
\hline $25 / 3 / 2018$ & 98.5 \\
\hline $26 / 3 / 2018$ & 97 \\
\hline $27 / 3 / 2018$ & 95 \\
\hline $28 / 3 / 2018$ & 96 \\
\hline $29 / 3 / 2018$ & 95.5 \\
\hline $30 / 3 / 2018$ & 93 \\
\hline $31 / 3 / 2018$ & 92.5 \\
\hline $1 / 4 / 2018$ & 91.5 \\
\hline $2 / 4 / 2018$ & 90 \\
\hline $3 / 4 / 2018$ & 91 \\
\hline $4 / 4 / 2018$ & 89.5 \\
\hline $5 / 4 / 2018$ & 88 \\
\hline $6 / 4 / 2018$ & 87 \\
\hline $7 / 4 / 2018$ & 85 \\
\hline $8 / 4 / 2018$ & 83.5 \\
\hline $9 / 4 / 2018$ & 81 \\
\hline $10 / 4 / 2018$ & 80 \\
\hline $11 / 4 / 2018$ & 78.5 \\
\hline $12 / 4 / 2018$ & 77 \\
\hline $13 / 4 / 2018$ & 77.5 \\
\hline $14 / 4 / 2018$ & 77 \\
\hline
\end{tabular}

Table 2: Pedal Edema Assessment

\begin{tabular}{|c|c|c|}
\hline Date & \begin{tabular}{|l|} 
Just below Knee \\
\end{tabular} & Just Above Ankle \\
\hline $23 / 3 / 2018$ & Rt $29.5 \mathrm{~cm}$, Lt $30 \mathrm{~cm}$ & Rt $27.5 \mathrm{~cm}, \mathrm{Lt} 26 \mathrm{~cm}$ \\
\hline $24 / 3 / 2018$ & Rt $29.5 \mathrm{~cm}, \mathrm{Lt} 30 \mathrm{~cm}$ & Rt $27 \mathrm{~cm}, \mathrm{Lt} 26 \mathrm{~cm}$ \\
\hline $25 / 3 / 2018$ & Rt $29 \mathrm{~cm}$, Lt $29.5 \mathrm{~cm}$ & Rt $26.5 \mathrm{~cm}, \mathrm{Lt} 25.5 \mathrm{~cm}$ \\
\hline $26 / 3 / 2018$ & Rt $28.5 \mathrm{~cm}, \mathrm{Lt} 29.5 \mathrm{~cm}$ & Rt $26.5 \mathrm{~cm}, \mathrm{Lt} 25 \mathrm{~cm}$ \\
\hline $27 / 3 / 2018$ & Rt $28 \mathrm{~cm}$, Lt $29 \mathrm{~cm}$ & Rt $26 \mathrm{~cm}, \mathrm{Lt} 24.5 \mathrm{~cm}$ \\
\hline $28 / 3 / 2018$ & Rt $27.5 \mathrm{~cm}$, Lt $29 \mathrm{~cm}$ & Rt $25.5 \mathrm{~cm}$, Lt $24.5 \mathrm{~cm}$ \\
\hline 29/3/2018 & Rt $27 \mathrm{~cm}$, Lt $28.5 \mathrm{~cm}$ & Rt $25 \mathrm{~cm}, \mathrm{Lt} 23 \mathrm{~cm}$ \\
\hline $30 / 3 / 2018$ & Rt $26.5 \mathrm{~cm}$, Lt $28.5 \mathrm{~cm}$ & Rt $24.5 \mathrm{~cm}, \mathrm{Lt} 22.5 \mathrm{~cm}$ \\
\hline $31 / 3 / 2018$ & Rt $26.5 \mathrm{~cm}$, Lt $28 \mathrm{~cm}$ & Rt $24 \mathrm{~cm}, \mathrm{Lt} 22 \mathrm{~cm}$ \\
\hline $1 / 4 / 2018$ & Rt $26 \mathrm{~cm}, \mathrm{Lt} 27.5 \mathrm{~cm}$ & Rt $23.5 \mathrm{~cm}$, Lt $21.5 \mathrm{~cm}$ \\
\hline $2 / 4 / 2018$ & Rt $25.5 \mathrm{~cm}, \mathrm{Lt} 27 \mathrm{~cm}$ & Rt $23 \mathrm{~cm}, \mathrm{Lt} 21 \mathrm{~cm}$ \\
\hline $3 / 4 / 2018$ & Rt $24.5 \mathrm{~cm}, \mathrm{Lt} 27 \mathrm{~cm}$ & Rt $22.5 \mathrm{~cm}, \mathrm{Lt} 20.5 \mathrm{~cm}$ \\
\hline $4 / 4 / 2018$ & Rt $24 \mathrm{~cm}, \mathrm{Lt} 26.5 \mathrm{~cm}$ & Rt $22.5 \mathrm{~cm}, \mathrm{Lt} 20 \mathrm{~cm}$ \\
\hline $5 / 4 / 2018$ & Rt $23.5 \mathrm{~cm}, \mathrm{Lt} 26 \mathrm{~cm}$ & Rt $22 \mathrm{~cm}$, Lt $20 \mathrm{~cm}$ \\
\hline $6 / 4 / 2018$ & Rt $23.5 \mathrm{~cm}, \mathrm{Lt} 26 \mathrm{~cm}$ & Rt $21.5 \mathrm{~cm}$, Lt $19.5 \mathrm{~cm}$ \\
\hline $7 / 4 / 2018$ & Rt $23 \mathrm{~cm}$, Lt $25.5 \mathrm{~cm}$ & Rt $21.5 \mathrm{~cm}$, Lt $19.5 \mathrm{~cm}$ \\
\hline $8 / 4 / 2018$ & Rt $23 \mathrm{~cm}, \mathrm{Lt} 25.5 \mathrm{~cm}$ & Rt $20 \mathrm{~cm}$, Lt $19 \mathrm{~cm}$ \\
\hline $9 / 4 / 2018$ & Rt $22.7 \mathrm{~cm}$, Lt $25 \mathrm{~cm}$ & Rt $20.5 \mathrm{~cm}$, Lt $18.5 \mathrm{~cm}$ \\
\hline $10 / 4 / 2018$ & Rt $22.5 \mathrm{~cm}, \mathrm{Lt} 24.5 \mathrm{~cm}$ & Rt $20.5 \mathrm{~cm}$, Lt $18.5 \mathrm{~cm}$ \\
\hline $11 / 4 / 2018$ & Rt $22 \mathrm{~cm}, \mathrm{Lt} 24 \mathrm{~cm}$ & Rt $20 \mathrm{~cm}, \mathrm{Lt} 18 \mathrm{~cm}$ \\
\hline $12 / 4 / 2018$ & Rt $22 \mathrm{~cm}, \mathrm{Lt} 23.5 \mathrm{~cm}$ & Rt $20 \mathrm{~cm}, \mathrm{Lt} 18 \mathrm{~cm}$ \\
\hline
\end{tabular}




\begin{tabular}{|lll|}
\hline $13 / 4 / 2018$ & Rt $22 \mathrm{~cm}$, Lt $23.5 \mathrm{~cm}$ & Rt $19.5 \mathrm{~cm}$, Lt $17.5 \mathrm{~cm}$ \\
\hline $14 / 4 / 2018$ & Rt $22 \mathrm{~cm}$, Lt $23 \mathrm{~cm}$ & Rt $19 \mathrm{~cm}, \mathrm{Lt} 17 \mathrm{~cm}$ \\
\hline
\end{tabular}

Table 3: Investigations Before and After Treatment

\begin{tabular}{|c|c|c|}
\hline Test & Before Treatment & After Treatment \\
\hline Hb\% & $6.2 \mathrm{gm} / \mathrm{dl}$ & $8.2 \mathrm{gm} / \mathrm{dl}$ \\
\hline $\begin{array}{l}\text { WBC } \\
\text { count }\end{array}$ & $15,500 / \mathrm{cmm}$ & $11000 / \mathrm{cmm}$ \\
\hline $\begin{array}{l}\text { Platelet } \\
\text { count }\end{array}$ & $1,66,000$ & $1,73,000$ \\
\hline $\begin{array}{l}\text { Urine } \\
\text { analysis }\end{array}$ & Albumin-Present. Pus cells- Plenty/hpf & $\begin{array}{l}\text { Albumin absent. Pus cells- } \\
\text { occasional. }\end{array}$ \\
\hline $\begin{array}{l}\text { Liver } \\
\text { Function } \\
\text { Test }\end{array}$ & $\begin{array}{l}\text { Sr. BilirubinTotal- } 2.43 \mathrm{mg} / \mathrm{dl} \text {, } \\
\text { Indirect- 1.30Direct-1.13 } \\
\text { SGOT -83 IU/LitSGPT -52 IU/Lit Total } \\
\text { Protein- } 7.9 \mathrm{~g} / \mathrm{dl} \\
\text { Albumin- } 3.8 \mathrm{~g} / \mathrm{dlGlobulin} \text { (calculated) } \\
4.10 \mathrm{~g} / \mathrm{dl} \\
\text { Alkaline phosphate-132 u/l }\end{array}$ & $\begin{array}{l}\text { Sr. Bilirubin Total- } 1.6 \mathrm{mg} / \mathrm{dl} \text {, } \\
\text { Indirect- } 1.19 \text { Direct- } 0.41 \\
\text { SGOT -80 IU/Lit SGPT }-51 \\
\text { IU/Lit Total Protein- } 7.9 \mathrm{~g} / \mathrm{dl} \\
\text { Albumin- } 3.9 \mathrm{~g} / \mathrm{dl} \\
\text { Globulin(calculated) } 4.00 \mathrm{~g} / \mathrm{dl} \\
\text { Alkaline phosphate- } 129 \mathrm{u} / 1\end{array}$ \\
\hline $\begin{array}{l}\text { Renal } \\
\text { Function } \\
\text { test }\end{array}$ & $\begin{array}{l}\text { Blood Urea-32 mg/dl, Blood Urea nitrogen- } \\
15.24 \mathrm{mg} / \mathrm{dl} \text {, Sr. Creatinine- } 1.1 \mathrm{mg} / \mathrm{dl}\end{array}$ & $\begin{array}{l}\text { Blood Urea-33 mg/dl, Blood } \\
\text { Urea nitrogen- } 15.71 \mathrm{mg} / \mathrm{dl}, \mathrm{Sr} \text {. } \\
\text { Creatinine- } 0.96 \mathrm{mg} / \mathrm{dl}\end{array}$ \\
\hline $\begin{array}{l}\text { USG } \\
\text { abdomen }\end{array}$ & $\begin{array}{l}\text { Borderline Hepatomegaly with } \\
\text { Mild splenomegaly. Raise reflectivity of } \\
\text { Liver with coarse parenchymal and gross } \\
\text { surfacenodularity. Gross } \\
\text { ascites. }\end{array}$ & $\begin{array}{l}\text { Liver Grossly normal in size with } \\
\text { mild sleenomegaly. Moderate } \\
\text { ascites. }\end{array}$ \\
\hline
\end{tabular}

\section{References}

[1] Prof. Ravidatta Tripathi, 2007 Editor Charaka Samhita, Chaukhamba Prakashana, Varanasi, siddhisthana $1 / 17,879$.

[2] KajariaDivya, Tripathi J. S., Tiwari S. K., Utilization of panchakarma in health care: Preventive, Nutritive and Curative treatment of disease, jpsi 2(5) septocto.2013 1-5.

[3] Antarkar D. S., Vaidya A. B., Joshi J. C.: A double blind clinical trial of Arogyavardhini an ayurvedic drug in acute viral Hepatitis. Ind.J.med.Res72 588-593.

[4] http://www.wikipedia.org/wiki/cow urine.

[5] Bhavamishra, Bhavaprakasha with Vidyotini Hindi Commentary, edited in Hindi by Sri Brahma Shankar Mishra and Sri Rupalalji Vaisya,11th Ed Varanasi Chaukhamba Sanskrit Sansthan;2004.

[6] Longo, Fauci etc., Harrison's Principles of Internal Medicine 18th Ed REFERENCES

*Corresponding author.

E-mail address: albertphd1986@gmail.com 